\title{
Tringa Temminckii.
}

Zwar häufig Ende Juli bis September und April und Mai an verschiedenen Orten gesehen und gar manche erlegt, doch waren sie nie, wie Andere angeben, in geschlossenen Schwärmen oder gar unter Schwärmen anderer Arten. Sie hielten sich ausnahmslos abgesondert und $\mathrm{zu}$ nur wenigen.

Thalassidroma pelagica.

Im November 1868 zum ersten Male auf Borkum erhalten.

(Diomedea exulans.)

Es dürfte erwähnenswerth sein, dass ein Flügel davon im Frühjahr 1868 auf Borkum angespült wurde. Selbstredend ist dadurch durchaus nicht bewiesen, dass dieser betreffende Albatros je die Nordsee lebend erblickt habe.

\section{Mormon fratercula.}

Wird fast nur in sehr kalten Wintern bemerkt. Todte werden auch sehr selten angespült.

\section{Mergulus alle.}

Habe ich häufiger als vorigen erhalten. Uebrigens auch nur in kalten Wintern.

Podiceps cristatus.

" rubricollis.

" arcticus sive cornutus.

" nigricollis sive auritus Naum.

" minor.

Alle durchaus nicht so selten auf den Inseln und im Marschlande.

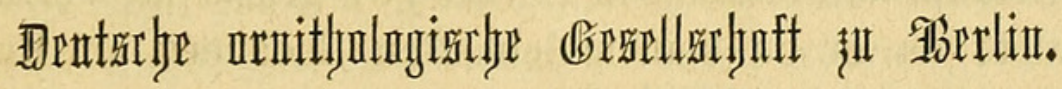

\section{Protokoll der VII. Monats-Sitzung.}

Verhandelt Berlin, Montag den 5. October 1868, im Bureau des Aquariums.

Vorsitzender: Herr C. Bolle.

Der Geschäftsführer berichtet über die für die Jahresversammlung getroffenen Vorbereitungen.

Hierauf nahm den grösseren Theil der Sitzung, welche als Vorversammlung zu der in den beiden folgenden Tagen statthabenden Jahresversammlung diente, die Besprechung und Feststellung des Programms ein, woran sich dann bis zum Schlusse der Sitzung eine zwanglose Unterhaltung über verschiedene ornithologische Gegenstände schloss. 


\section{Protokoll der Ersten JahresversammIung.}

Verhandelt Berlin, Dienstag den 6. und Mittwoch den 7. October 1868 im Gasthof zum ,Landhause“.

Zur Jahresversammlung anwesend waren:

Aus Berlin die Herren Freese, Golz, Cabanis, Bolle, Russ, Tichy, v. Assel, Reichenow, Blümel und Mosson.

Von auswärtigen Mitgliedern: die Herren Lühder aus Greifswald und Jablonski aus Gollmütz.

Als Gäste: Die Herren Dr. Krüper aus Griechenland, Dr. E. Baldamus und Hr. Schlüter aus Halle, und Hr. F. Erman aus Berlin.

Vorsitzender: Hr. Cabanis. Protokollführer: Hr. Bolle.

Dienstag, Vormittags 10 Uhr:

Der Vorsitzende eröffnet die Sitzung mit Begrüssung der zur Versammlung gekommenen auswärtigen Mitglieder und Gäste, und ersucht letztere an den Verhandlungen sich zu betheiligen.

Es kommen die an die Jahresversammlung eingegangenen Zuschriften zum Vortrag.

Herr Eugen v. Homeyer sendet der Versammlung seinen Gruss und bedauert, am Erscheinen verhindert zu sein, die bevorstehende Vollendung der Eisenbahn nach Stolp werde ihm den Besuch von Berlin in der Folge erleichtern.

Herr Hauptmann Alex. von Ho m e y er grüsst die Versammlung und sendet, da er zu seinem lebhaften Bedauern am Erscheinen verhindert ist, einige Mittheilungen ein, welche besonders abgedruckt werden.

Herr Hofrath Dr. v. Heuglin grüsst und sendet die Fortsetzung seiner monographischen Artikel im Journal mit der Anzeige, dass er die Resultate seiner Beobachtungen über die Vögel N.-O.-Afrikas als Bilderwerk demnächst zu veröffentlichen beginnen werde.

Hr. Dr. Finsch, zugleich im Namen des Hrn. Dr. Hartla ub, schreibt: „Ich hoffte, zu unserer Versammlung, der wir leider nicht beiwohnen können, den 2. Band der Papageien einsenden zu können, es geht zu meinem Bedauern indess nicht, da das Werk bis dahin noch nicht fertig wird. Jedenfalls seien Sie so gut, der Versammlung unsere besten Grüsse auszusprechen, 
wobei Sie erwähnen wollen, dass unser westafrikanischer Sammler, Sinte $\mathrm{n}$ is, ein Schlesier, glücklich in Accra angelangt ist und seine Thätigkeit begonnen hat; es ist doch immerhin von Interesse, dass ein neuer deutscher Sammler draussen thätig ist und auf einem Gebiete, welches noch Manches verspricht."

Der Geschäftsführer erwähnt hierauf eines zweiten Briefes des Hrn. Dr. Finsch, und macht die folgende Mittheilung: In einem zu Münster gedruckten und unter Kreuzband versandten „Berichte" über eine Anfangs Juni d. J. zu Kiel abgehaltene Ornithologen-Versammlung befinden sich einige missliebige Aeusserungen über die Weise, auf welche das „Comité" (GründungsAusschuss) unserer Gesellschaft gebildet sei. Der Geschäftsführer soll die Namen einiger Vorstandsmitglieder ohne deren Zustimmung beigefügt haben, und zwar wird diese Verdächtigung auf briefliche Mittheilungen solcher Mitglieder begründet. Da es Zweifel in die Ehrenhaftigkeit eines Vorstandsmitgliedes setzen hiesse, wenn man annehmen wollte, dass es mit einer Beschwerde nicht zunächst an sein eigenes Collegium, sondern, ,in sein eigen Fleisch schneidend," durch trübe Kanäle an die Oeffentlichkeit gehe, da ferner bis heut keinerlei Beschwerde beim Vorstande eingegangen sei, die gegentheiligen schriftlichen Beweise aber, wozu auch der Brief des Herrn Dr. Finsch zähle, bei den Akten der Gesellschaft sich befänden und für Jedermann einzusehen seien - so könne und dürfe die ganze Angelegenheit unsere Gesellschaft richt weiter berühren. In derselben Lage befinde sich der Geschäftsführer, und sei dieser auch nicht entfernt gewillt, durch weiteres Eingehen auf eine indiscrete Angelegenheit Dritter, die mit Förderung der Ornithologie nichts $\mathrm{zu}$ thun habe, die Zeit und die Berichte unserer Sitzungen auszufüllen. Es sei dies obenein gegen unsere Statuten, deren richtige Würdigung mit der Zeit selbst den Anschluss der wenigen noch scheelsehenden ornithologischen Partikularisten Deutschlands an die in Berlin, mit der Aussicht auf Dauer, begründete Gesellschaft hoffen lasse.

Herr Dr. Anton Fritsch aus Prag sendet der Gesellschaft seinen Gruss, begleitet von Notizen über Vorkommnisse in Böhmen, welche besonders abgedruckt werden.

Herr A. v. Pelzeln hat die soeben erschienene II. Abtheilung seines höchst schätzenswerthen Werkes über Brasilien eingesandt, dasselbe wird vom Vorsitzenden ausführlicher besprochen. Der Titel ist: „Zur Ornithologie Brasiliens. Resultate von Johann Natterer's 
Reisen in den Jahren 1817 bis 1835." Wien, 8 . Ein Buch, welches für jede ornithologische Sammlung unentbehrlich und Jedem, der sich für südamerikanische Ornithologie interessirt, zu empfehlen ist. Die selbst für den Eingeweihteren überraschend grosse Zahl der neuen, daselbst beschriebenen Arten giebt Zeugniss von dem rastlosen Eifer Natterer's, welcher während 17 Jahren das grosse Kaiserreich Brasilien gleichsam von Station zu Station umkreiste. Im Süden beginnend, von dort nach Westen gehend und dann in nördlicher Richtung fortschreitend, sammelte derselbe die Repräsentanten des südlichen, centralen, bolivisch brasilischen, columbischbrasilischen, guianisch-brasilischen Faunengebietes und schloss mit dem des unteren Amazonenstromes. Eine der I. Abtheilung beigegebene Karte dient zur Erläuterung. Die III. Abtheilung, der aber wohl noch einige weitere folgen dürften, wird Anfangs des nächsten Jahres erscheinen. Herr v. Pelzeln hat mit dem Werke nicht nur dem um die Ornithologie Brasiliens hochverdienten Joh. Natterer ein würdiges Denkmal gesetzt, sondern durch Herausgabe desselben sich der dankenden Anerkennung aller Ornithologen von Fach versichert.

Herr A. Kuwert hat eine Kiste mit seltneren exotischen Vogelbälgen zur Ansicht und Bestimmung, sowie Notizen über das jetzige Vorkommen von Nucifraga caryocatactes in Ostpreussen eingesandt. Letztere werden besonders abgedruckt, und bemerkt der Vorsitzende hierzu, dass auch hier in der Mark der Vogel in den letzten Wochen mehrfach bemerkt und erlegt worden sei.

Herr G. Schneider in Basel sendet seinen Gruss, verheisst Beiträge für das Journal und hofft, dass es ihm gelingen werde, lebende Kalanderlerchen für hiesige Liebhaber senden zu können.

Eine an die Versammlung eingegangene Anfrage wegen eines Heilmittels für eine angegebene Krankheit eines Stubenvogels wird besprochen, und Herr Bolle übernimmt im Namen der Gesellschaft die Beantwortung.

Herr Carl Mayrhofer, Präparator in Augsburg, H. 342, empfiehlt sich als Präparator an einer Staats- oder Privatsammlung oder auch als solcher bei Reisen und Expeditionen, was hiermit zur Kenntniss gebracht werden soll.

Es folgt nunmehr die Mittheilung, dass der Gesellschaft bis jetzt 46 Mitglieder beigetreten seien. Das Verzeichniss derselben wird verlesen und soll am Schlusse des Jahres abgedruckt werden.

Herr C. Russ hält einen Vortrag über die von ihm betriebene 
Zucht fremdländischer Vögel, welcher im Journale ausführlich abgedruckt werden wird.

Auf Einladung des Herrn Russ begiebt sich die Versammlung hierauf in die Wohnung desselben, um seine "Vogelstube“ in Augenschein zu nehmen. Diese gewährte einen überraschend freundlichen Anblick, und allgemein wurde dem Besitzer sowohl in Bezug auf die äussere Anordnung wie auf die zweckmässige Pflege und die erzielten günstigen Resultate die vollste Anerkennung der Versammlung zu Theil.

Hierauf kehrte man in das Versammlungslokal zurück, woselbst eine inzwischen festlich geordnete Mittagstafel die Theilnehmer in traulichem Beisammensein bis gegen Abend vereint hielt.

Mittwoch, Vormittags 10 Uhr.

Nach vorgängiger Besichtigung der ornithologischen Abtheilung des zoologischen Museums, bei welcher mitgebrachte Bälge bestimmt und über manche zweifelhafte Frage Aufschluss erlangt wurde, begann die zweite Sitzung.

Herr Ca ban is berichtete über die ornithologische Ausbeute der Reisen des Baron v. d. Decken in Ost-Afrika. Nachdem der Tod dem Forschungsdrange des bekannten Reisenden mitten in seiner Thätigkeit im Innern Ost-Afrikas ein zu frühzeitiges Ziel gesetzt hatte, beschloss die Familie desselben, vertreten durch die Frau F ürstin Pless, die Veröffentlichung des Reisewerkes und beauftragte mit der Herausgabe den Herrn Dr. Kersten, einen Reisegefährten des verstorbenen Barons. Mit anerkennenswerther Liberalität wurden die hinterbliebenen zoologischen Gegenstände dem Königlichen zoologischen Museum zur Aufbewahrung und wissenschaftlichen Bearbeitung einverleibt. In Folge dessen überkam dem Vortragenden die Pflicht der Bearbeitung der ornithologischen Abtheilung. Es wurde keine Mühe gescheut, das an verschiedenen Orten zerstreute, theils durch ungeschickte Privatpräparatoren verwahrloste, theils in Weingeist aufbewahrte Material $\mathrm{zu}$ sammeln und $\mathrm{zu}$ einem bleibenden Andenken an den opfermuthigen deutschen Reisenden zu gestalten. Nach sorgfältiger Prüfung und Benutzung eines jeden vorhandenen Stückes ergab sich das befriedigende Resultat von 126 Vogelarten, worunter 20 als für die Wissenschaft neu anzusprechen waren. Der wünschenswerthen naturgetreuen bildlichen Darstellung hat sich Herr Th. v. Heuglin in dankenswerther Weise unterzogen 
Ein Abdruck der Bearbeitung (Bd. III, S. 19-52) und die zu derselben gefertigten 18 Tafeln werden hierauf vorgelegt und die neuen Arten kurz charakterisirt:

1. Turdus Deckeni, Taf. I, kleiner und dunkler als T. Cabanisi Bp., Bauchmitte und Weichen matt rostroth, untere Flügeldecken intensiver rostroth. Ganze Länge 9".

2. Bessornis intermedia, Taf. XII, kleiner und mit dunklerer Unterseite als Heuglini, der weisse Superciliarstreif schmäler aber länger.

7. Cisticola haematocephala, Taf. II, Fig. 2, etwas kleiner als lugubris Rüpp. Oberkopf nicht intensiv rothbraun, sondern durch einen olivenfarbenen Anflug gedämpft, Brustseiten und Oberscbwanzdecken nicht schwarz gefleckt; Grundfarbe der Steuerfedern gr a u braun mit schwarzem Fleck vor den weisslichen Spitzen.

8. Drymoeca tenella, Taf. II, Fig. 1, von mystacea Rüpp. durch grösseren Schnabel, kürzere Flügel und Schwanz und durch graubräunliche Oberseite, von superciliaris durch nicht rostrothe Weichen unterschieden.

11. Prionops graculinus, Taf. III, eine schöne Art, durch gänzlichen Mangel einer weissen Flügelbinde von Retzii unterschieden. Ganze Länge $81 / 2 "$.

14. Dryoscopus leucopsis, kleiner als affinis, mit dickerem, breiterem Schnabel, Flügel ohne weisse Abzeichen, Zügel und Augengegend we is s, nicht schwarz.

15. Dryoscopus thamnophilus, Taf. VIII, in der Färbung an Thamnophilus ambiguus Sw. erinnernd. G. L. $6{ }^{1 / 2}$ ".

17. Rhynchastatus lugubris, Taf. VII, fast einfarbig schwärzlich schiefergrau, dem funebris (Hartl.) sehr ähnlich, aber kleiner. G. L. $61^{1}{ }^{\prime \prime}$.

20. Lanius caudatus, Taf. V, mit entwickelten Stirnfedern und sehr langem Schwanze; Oberseite schwarzbraun, Rücken graubraun, Bürzel weiss; Schwanz schwarz, mit weisser Basis; Flügel mit

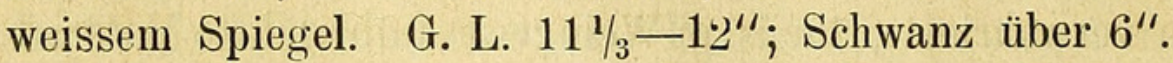

28. Crithagra chloropsis,*) Taf. IX, Vorderkopf, Kopfseiten und Unterseite einfarbig gelb, ohne dunkle Abzeichen; Oberseite grün mit dunkleren Schaftstrichen. G. L. $5^{1} / 4^{\prime \prime}$.

*) Der Name dieser neuen Art muss in Crith. imberbis umgeändert werden, da es leider zu spät bemerkt wurde, dass Bonaparte, welcher das Genus anders deutet, schon einen amerikanischen Vogel so benannt hatte. 
29. Calyphantria comorensis, Taf. X, von C. Algondae (Schleg.) verschieden durch schwarze Zügel, durch das an der Unterseite weiter hinabgehende Roth, durch weissgerandete Flügeldecken; Bürzel und Steiss mit Roth. Von Mayotte.

32. Textor intermedius, Taf. XI, von erythrorhynchus durch die nicht weisse Innenseite des Flügels, welcher daselbst nur einen weissgrauen Fleck zeigt, und durch weniger ausgebreitete Ränder an der Oberseite des Flügels verschieden. G. L. $91 / 2{ }^{\prime \prime}$.

58. Rhynchaceros (Tockus!) Deckeni, Taf. VI, von den verwandten kleinen Arten durch die dunklere, schwärzliche Oberseite und die nicht so bunten Flügeldecken unterschieden. Kopfseiten, Hals, Unterseite und Mittellinie am Rücken weiss. G. L. 17"; Fl. $6{ }^{3}{ }_{4} "$; Schw. $73 / 4 "$.

72. Melierax poliopterus, kleiner als musicus und mit einfarbig weissen oberen und unter en Schwanzdecken; sämmtliche Flügeldecken sowie die Schwingen zweiter Ordnung einfarbig aschgrau, ohne vermiculirte Querzeichnung. G. L. $20^{1 / 2}{ }^{\prime \prime}$; Fl. $12^{1 / 2}{ }^{\prime \prime}$; Schw. $9^{\prime \prime}$; Lauf $3^{1 / 2}{ }^{\prime \prime}$.

84. Pterocles decoratus, Taf. XIII, eine schöne, schon allein durch die Kopfzeichnung charakterisirte Art. Stirn, Gegend um den Schnabel, Stelle hinter dem Auge und Kehle schwarz; Fleck auf der Stirnmitte, Einfassung des Schwarz am Vorderkopf und Superciliarstreif weiss. G. L. 9"; Fl. $6 \frac{1}{2}{ }^{\prime \prime}$.

85. Pternistes infuscatus, Taf. XIV, dunkler als mubricollis Rüpp. und durch abweichende Zeichnung und verschiedene Farbe der oberen und namentlich der unteren Schwanzdecken verschieden. Letztere sind schwarzbraun, an der Spitzenhälfte breit weisslich gerandet. G. L. $16^{1} / 4^{\prime \prime}$; Fl. $8^{\prime \prime}$; Lauf $2^{1 / 3} 3^{\prime \prime}$.

89. Otis (Lissotis) maculipennis, Taf. XV, von melanogastra verschieden durch nicht quergewellten, sondern längsgestreiften Hals, die Mitte der Federn hell, seitlich schwarzbraun eingefasst; der Rücken sbunter, die Spitzenhälfte der Federn schwarz mit einem V-artigen, hellen Zeichen versehen; die Schwingen schwarz, die zweite an der Innenfahne, die folgenden auf beiden Fahnen weiss quergefleckt. G. L. $28^{\prime \prime}$; Fl. $12^{1 / 4}{ }^{\prime \prime}$; Lauf $4^{3} / 4^{\prime \prime}$.

94. Oedicnemus vermiculatus, Taf. XVI, eine dem senegalensis ähnliche, kleinere Art. Schnabel schwärzlich. Oberseite ausser der schwärzlichen Schaftstrichelung noch zickzackförmig dunkelbraun quergezeichnet; unter dem schwärzlichen oberen Flügelrande 
ein weisser Längsstreifen, der aber unterhalb nicht wie bei crepitans wiederum schwarz eingefasst ist. G. L. $14^{\prime \prime}$; Fl. $71_{2}{ }^{\prime \prime}$; Lauf $2^{3 / 4}{ }^{\prime \prime}$.

107. Ardea (Herodias) procerula, ein weisser Reiher, der garzetta sehr ähnlich, diese aber in den Grössenverhältnissen sehr überragend. Schn. v. Mundw. $4 \frac{1}{2}{ }^{\prime \prime}$, v. d. Stirn $4^{\prime \prime}$; Flügel $11^{1 / 2} \mathbf{2}^{\prime \prime}$; Schw. 4" $8^{\prime \prime \prime}$; Lauf $4^{\prime \prime} 5^{\prime \prime \prime}$; Mittelzehe ohne Kralle $2^{\prime \prime} 7^{\prime \prime \prime}$.

108. Ardea (Herodias) cineracea, Taf. XVII, heller grau als schistacea, und wie diese mit weisser Kehle; Schnabel aber zierlicher und fast schwarz, alle sonstigen Verhältnisse kleiner.

Für das Weitere wird auf das baldigst erscheinende Reise werk verwiesen und hervorgehoben, dass dasselbe sowohl nach seinem wissenschaftlichen Inhalte als seiner vorzüglichen Ausstattung, mithin in jeder Beziehung ein den deutschen Reisenden ehrendes und dessen Andenken dauernd begründendes Werk sein werde. Die Hinterbliebenen hätten nicht nur kein Opfer gescheut, dasselbe zu einer gediegenen Erscheinung zu machen, sondern auch über die Grenzen des eigentlichen Reisewerkes hinaus wissenschaftliche Zwecke bereitwilligst unterstützt und gefördert. Dies sei namentlich auch für die Ornithologie geschehen, und gereiche es dem V o r trag enden zu besonderem Vergnügen, schon jetzt mit wärmsten Danke erwähnen zu können, dass die Frau Fürstin Pless den Herausgeber des Reisewerkes, Herrn Dr. O. K ersten ermächtigt habe, mehrere ornithologische Tafeln des Reisewerkes in hinreichender Anzahl verfertigen zu lassen, damit dieselben dem Journal für Ornithologie kostenfrei einverleibt werden können.

Nach brieflicher freundlicher Mittheilung des Herrn Dr. Kers ten werden dem Herausgeber des Journals 6 bis 8 Tafeln zur Verfügung gestellt werden, und zwar sollen zwei derselben, als Probe des von Decken'schen Reisewerkes, den nächsten Heften des Journals beigegeben werden, die übrigen selbstverständlich erst nach dem Erscheinen der betreffenden Abtheilung des Reisewerkes. Der Vortragende knüpft an diese dem Journal gewährte Förderung die Hoffnung, dass diese in England und Nordamerika in ähnlichen Fällen längst übliche schöne Sitte, nach dem Vorgange der Fra u $\mathrm{Fürstin} \mathrm{Pless}$ nunmehr auch in Deutschland heimisch werden möge.

Als wesentliche Förderung der Ornithologie, zu welcher das v. Decken'sche Reisewerk die Anregung und die Mittel gewährte, sei ferner die von den Herren Hartlaub und Finseh in Bearbeitung genommene vollständige Ornithologie $\mathrm{Ost}$ - Afrikas 
gebührend hervorzuheben, welche als IV. Band des v. D e c k e n'schen Reisewerkes erscheinen werde. Noch fehle in der ornithologischen Literatur eine Aufzählung aller bisher in Ost-Afrika überhaupt beobachteten Vogel-Arten, und die Zusammenstellung derselben von so ausnehmend sachkundiger Seite sei daher als ein für jeden Ornithologen höchst willkommenes Ereigniss schon im Voraus zu begrüssen. Den Verfassern seien die v. Decken'schen Originalexemplare sowie das ganze auf Ost-Afrika bezügliche ornithologische Material des Berliner Museums zur Benutzung gestellt worden, und da dieselben auch sonst keine Mühe scheuten, sich die Quellen für ostafrikanische Vögel allseitig zugänglich zu machen, so sei auf eine, jede Voraussicht übertreffende Reichhaltigkeit und Vollständigkeit mit Bestimmtheit zu zählen. Der betreffende Band sei bereits im Drucke und werde ohne Aufenthalt gefördert.

Den Schluss der Jahresversammlung bildete ein ausführlicher Vortrag des Herrn Bolle, welcher, anknüpfend an die neuerdings erfolgte Auffindung einer weiteren Abbildung des Dodo durch Ritter v. Frauenfeld, über die sonstigen neuen osteologischen Entdeckungen in Bezug auf diese ausgestorbene Vogelgruppe Mittheilung machte. (Wird im Journal ausführlich mitgetheilt werden.)

C. Bolle.

J. Caban is, Secr.

\section{Verzeichniss \\ der Mitglieder der Gesellsehaft.}

(Im ersten Jahre, 1868, beigetreten.)

\section{Vorstand :}

1. Cabanis, J., Dr., Secretär d. Gesellschaft, in Berlin.

2. Bodinus, Dr., Director d. Zoolog. Gartens in Cöln a. R.

3. Bolle, Carl, Dr., in Berlin.

4. Brehm, Alfr., Di., in Berlin.

5. Finseh, Otto, Dr., in Bremen.

6. Hartlaub, G., Dr., in Bremen.

7. Heine, F., Ober-Amtmann auf St. Burchard vor Halberstadt.

8. v. Heuglin, M. Th., Dr., Hotrath, in Ober-Türkheim, Würtemberg.

9. v. Ho meyer, Eug. F., Rittergutsbesitzer auf Warbelow, Pommern.

10. v. Homeyer, Alex., Hauptmann im 38. Füsilier-Reg. in Görlitz.

11. König, Richard, Freiherrr von und zu Warthausen, Würtemberg.

12. y. Pelzeln, Aug., Custos am k. k. Zoologischen Cabinet in Wien.

13. Golz, H., Dr., Rechtsanwalt und Notar, in Berlin.

14. T a u ber, Eduard, Fabrikbesitzer in Tückelhausen, Bayern.

15. S a chse, Carl, Baumeister, in Altenkirchen, Rheinprovinz. 


\section{$2 \mathrm{BHL}$ Biodiversity Heritage Library}

1868. "Protokoll der VII. Monats-Sitzung." Journal fu

r Ornithologie 16, 407-415. https://doi.org/10.1007/bf02261114.

View This Item Online: https://www.biodiversitylibrary.org/item/103094

DOI: https://doi.org/10.1007/bf02261114

Permalink: https://www.biodiversitylibrary.org/partpdf/142056

\section{Holding Institution}

Smithsonian Libraries

\section{Sponsored by}

Biodiversity Heritage Library

\section{Copyright \& Reuse}

Copyright Status: Public domain. The BHL considers that this work is no longer under copyright protection.

This document was created from content at the Biodiversity Heritage Library, the world's largest open access digital library for biodiversity literature and archives. Visit BHL at https://www.biodiversitylibrary.org. 